\title{
OPTIMIZING CONTAINER LOCATION FOR SELECTIVE COLLECTION OF URBAN SOLID WASTE
}

\author{
EVA BARRENA ${ }^{1}$, DAVID CANCA ${ }^{2}$, FRANCISCO A. ORTEGA ${ }^{3} \&$ RAMÓN PIEDRA-DE-LA-CUADRA ${ }^{2}$ \\ ${ }^{1}$ Universidad Pablo de Olavide, Spain \\ ${ }^{2}$ Higher Technical School of Engineering, Universidad de Sevilla, Spain \\ ${ }^{3}$ Higher Technical School of Architecture, Universidad de Sevilla, Spain
}

\begin{abstract}
Municipal solid waste management includes several functional phases such as waste generation, storage, collection, transportation, processing, recycling and disposal in a suitable landfill. Waste collection and transportation phases are closely related, since the deployment of containers along the city determine the vehicle fleet size required for picking up the collected waste into the containers and the design of efficient routes needed for that purpose. A mathematical model for the deployment of containers in a context of selective collection of urban solid waste, has been formulated in this work. A greedy algorithm of overflowing deviated to the immediate neighbourhood has also been developed to solve the proposed mathematical programming model. In order to evaluate the performance of the developed methodology, a computational experience has been carried out on an urban system inspired in a zone belonging to the area of Seville, Spain.
\end{abstract}

Keywords: separate collection of wastes, location of waste containers, collection routes.

\section{INTRODUCTION}

Municipal Solid Waste (MSW) is commonly defined as the waste generated in households, commercial establishments, institutions, and businesses. MSW includes used paper, discarded cans and bottles, food scraps, yard trimmings, and other items. Other garbage such as residues of industrial process, agricultural wastes, mining waste, and sewage sludge are not included in the terminology MSW. The main functional phases of MSW management are waste generation, storage, collection, transportation, processing, recycling and disposal in a suitable landfill [1].

Typically, collection costs represent $80-90 \%$ and $50-80 \%$ of municipal solid waste management budget in low income and middle income countries, respectively [2]. Therefore, waste collection and transportation problems are one of the most difficult operational problems when developing an integrated waste management system [3]. Eiselt and Marianov [4] provide a survey of 64 works that include applications throughout the world, where the main aspects of interest of the contributions have been summarized in a table (see [4]), by selecting country, technique, criteria, objectives and type or facility to be located.

Waste Management (WM) is focused on providing solid-waste collection services for residential, industrial, and commercial customers in metropolitan areas. Residential customers are generally people living in private homes, whereas commercial customers include strip malls, restaurants, and small office buildings. Additionally, the intrinsic nature of MW collection relates to the development of effective vehicle routing (VR) models that optimize the total traveling distance of vehicles, the environmental emission and the investment costs [5]. VR is a scheduled process that allows vehicles to load waste at gather sites and dump it at a landfill with the target being oriented by a single or multiple objectives [6]. Through a Route optimization for Waste management (WR), both the residential routing problem and the commercial routing problem settings can be solved.

In real scenarios, the waste collection system is distributed in a set of zones. Each zone has a starting and a ending nodes associated. These are used for vehicle routes and landfill 
points where one can deliver the garbage collected in the visited containers. A planning horizon must also be considered in order to schedule a sequence of services within its bounds. A succession of routes (one per day, belonging to the same or to different distribution zones and performed by the same vehicle along the planning horizon) is called circulation. Plans for determining the vehicle circulation in transportation networks are described in [7].

The problem of managing selective collection of waste containers inside historic centres can be performed in three sequential phases: first, the location of containers along the streets; then, the determination of the minimum fleet size required to perform all collecting services; and finally, a model devoted to identify the optimal routes, in terms of total and equilibrated number of kilometres travelled by the trucks, is required. Obviously, the result of the first phase (location of the containers) highly influences the procedure since this will determine the decision to be taken for the subsequent phases (route of collection vehicles and service programming).

The main contribution of this paper focuses on this first phase: the location of collecting facilities (waste containers), where facility-customer distances must be considered in the collecting design system, as well as other considerations such as the size of container groups, their capacities in accordance with the closest population and the installation cost of those containers in specific sites along the streets.

The remainder of this work is organized as follows. In Section 2, an optimization model for locating waste containers is presented. Subsequently, the formulation of this decision model, in addition to its corresponding resolution algorithm, is developed. A computational experience based on an application to a real case is implemented in Section 3. Finally, some conclusions are summarized in Section 4.

\section{MODEL FORMULATION}

We assume the following mathematical context, associated to the characteristics of the problem, that consists of a connected graph $G=(V, A)$, composed of a node set $V$ (portals) and an arc set $A$ (directed edges representing street sections). The arcs of set $A$ connect the nodes belonging to set $V$, so that the existence of a shortest path in terms of distance or travel time between each pair of points of $V$ is guaranteed. Let us suppose that set $V$ is composed of nodes where urban waste is generated (set $I$ ) and by points where it is possible to locate the containers to deposit them (set $J$ ). We also assume that the inclusion sequence $J \subset I \subset V$ is maintained.

Note that any node $i$ of $V$ located at the entrance gate of a building could be identified as a generating point of waste; in that case, node $i$ would belong to set $I$. Alternatively, node $i$ could simply be a feasible site along the street, where the waste container could temporarily be located (in that case, node $i$ would also belong to set $J$ ).

The following notation is used in our waste containers formulation:

- $\quad I$ : set of demand nodes $(i \in I)$. There is a population $p_{i}$ associated to each demand point $i \in I$.

- $J$ : set of possible location nodes to locate waste containers $(j \in J)$. There is an upper bound ( $\mathrm{cap}_{j}, j \in J$ ) in terms of capacity associated to each candidate point $j \in J$.

- $K$ : set of main types of solid waste generated in the urban area (for instance, cardboard, plastic, organic waste, scrap metal, etc.) $(k \in K)$.

Additionally, we assume a compensation cost $\beta_{j}^{k}>0$ associated with the economic value that the municipal cleaning company would be willing to pay to maintain a container of 
modality $k$ in node $j$ during the planning horizon. This means that the cleaning company should have previously negotiated a payment reduction with the inhabitants nearby node $j$, due to the inconveniences generated by permanently establishing the container of type $k$ close to their dwellings. So, if node $j$ is located in the public domain far from any building, the compensation cost could be considered 0 ; on the other hand, if the location of the container in the proximity of a house is technically unfeasible, this cost could be associated to an infinite value.

The parameters involved in our optimization model are the following:

- $\quad$ Each node $i$ has a known weight $w_{i}{ }^{k}$ (which can be identified with the amount of waste in $\mathrm{kg}$ or $\mathrm{dm}^{3}$ generated in node $i$ of modality $k$, i.e., organic material, glass, packaging or paper units) associated.

- The shortest distances between nodes of set $V$, along network $G$, have previously been determined and recorded in the matrix $D=\left(d_{i j}\right), d_{i j} \geq 0$. Inhabitants associated to node $i$ would experience a displacement cost (discomfort) $C_{i j}{ }^{k}$ when having to take their type $k$ waste to the container located at point $j$. This discomfort implicitly requires a restriction on travel distances. In practice, this restriction is modelled by the assignation of a feasible coverage radius from point $i$. A point $i$ can be considered covered by another point $j$ if the distance between them does not exceed a radius of displacement $R^{k}$.

Let us assume that each customer is willing to use any container, as long as a maximum walking distance from their residence to the assigned container is not exceeded. That container might not be the closest, but this must lie within a predefined radius. In our model, a portion of inhabitants associated with node $i$ could take their garbage to the container $j$ and another portion of the population of the same node would be willing to take out their garbage to another unfilled container $j$ that is not excessively distant. This solidary behavior of the clients would allow an efficient deployment of the containers in the area under analysis, reducing their total number and grouping them in the points of lowest cost.

Let $q_{i j}^{k} \in\{0,1\}$ be a binary expression that takes value 1 if the demand point $i$ can be covered by site $j$ by means of a container of modality $k$ (note that $q_{i j}^{k}=1$ implies that $d_{i j} \leq R^{k}$, and value 0 , otherwise). Additionally, let $N_{j}>0$ be an integer parameter that indicates the maximum number of containers that could be installed at location $j$. We assume that all containers are provided with the same capacity $Q$.

Moreover, the following variables are required in the model.

\subsection{Variables}

$y_{j}^{k} \quad$ Binary variable that takes value 1 , if container location $j$ is activated to collect type $k$ garbage, and value 0 , otherwise.

$n_{j}^{k} \quad$ Number of type $k$ containers to be installed at location $j$.

$x_{i j}^{k} \quad$ Percentage of type $k$ garbage that the client corresponding to node $i$ will deliver at location $j$.

The nature of the variables used in the model yields varied formulations to face different objectives. In our case, the following integer programming formulation determines the minimum number of container groups to be installed in the area under consideration. Note that the lower the number of garbage deposit points, the more efficient the collection procedure will be. 
2.2 Objective and constraints

$$
Z_{1} \equiv \operatorname{Min} \sum_{j \in J} \sum_{k \in K} \beta_{j}^{k} n_{j}^{k}
$$

subject to:

$$
\begin{gathered}
\sum_{j \in J} q_{i j}^{k} y_{j}^{k} \geq 1, \quad i \in I, \quad k \in K, \\
\sum_{j \in J} q_{i j}^{k} x_{i j}^{k}=1, \quad i \in I, \quad k \in K, \\
x_{i j}^{k} \leq y_{j}^{k}, \quad i \in I, \quad j \in J, \quad k \in K, \\
y_{j}^{k} \leq n_{j}^{k}, \quad j \in J, \quad k \in K, \\
\sum_{i \in I} w_{i}^{k} x_{i j}^{k} \leq Q \cdot n_{j}^{k} \quad j \in J, \quad k \in K, \\
\sum_{k \in K} n_{j}^{k} \leq N_{j} \quad j \in J, \\
y_{j}^{k} \in\{0,1\}, \quad n_{j}^{k} \in Z^{+}, \quad x_{i j}^{k} \geq 0 .
\end{gathered}
$$

The objective function (1) minimizes the cost of containers that should be installed. Note that when radii $R^{k}$ decrease, the number of containers that can be grouped in the same location will then increase. Constraints (2) ensure that all demand is covered by the set of locations to be determined. Constraints (3) establish that the sum of coverage percentages for every demand point from the container must be equal to 1 . Constraints (4) imply that if a location is activated, then at least one container must be installed at it. Constraints (5) guarantee that if a location is not activated, then no demand point can be covered by it. Constraints (6) imply that demand points that may be served from location $j$ cannot exceed its capacity. Constraints (7) establish an upper bound on the number of containers that can be located at each site. Constraints (8) indicate the nature of the variables used in the model.

Maintaining the above described constraints, an additional criterion, consisting of minimizing user travel costs, can be incorporated into the objective by combining it with the previously considered minimization of costs in the deployment of the containers. The expression that follows formulates this purpose:

$$
z_{2} \equiv \operatorname{Min} \sum_{i \in I} \sum_{j \in J} \sum_{k \in K} C_{i j}^{k} y_{j}^{k}+\sum_{j \in J} \sum_{k \in K} \beta_{j}^{k} n_{j}^{k} .
$$

Both models (1) $-(8)$ and (1') $-(8)$ are of combinatorial nature and can be considered as instances of a Partial Set Covering problem [8]. The partial set covering model is NP-hard since it is a generalization of the traditional location set covering problem, which is NP-hard. In [9], the problem is discussed in detail and its complexity is proved. This fact justifies the use of algorithms that provide a good heuristic solution.

A model similar to the one previously proposed has been investigated in [10], in which heuristics were designed in a computationally feasible way and consistent with the approach. Tests carried out on randomly generated data have shown that a simple heuristic of 
Overflowing Deviated to Immediate Neighbourhood (ODIN) yields the best results if the inter-location spacing between adjacent containers is not excessively large. Taking these precedents into account, we propose the heuristic ODIN for solving our optimization model in order to determine the most effective deployment of waste containers along the street network.

\section{Heuristic ODIN}

1. Sort the points that generate urban waste according to their production, from highest to lowest levels and re-label them.

2. While there exists a generator point $i$ whose collection requirement exceed the established upper limit $N_{i}$; i.e. $\sum_{k \in K} n_{i}^{k} \geq N_{i}$ do

1.1 Identify the set of nodes $\operatorname{Prox}(i ; k)$ whose distances to node $i$ are less than $R^{k}$ (excluding node $i$ )

1.2 Sort the nodes $j \in \operatorname{Prox}(i ; k)$ from the lowest to the highest levels according to ascending values of $\beta_{j}^{k}$

1.3 For each $j \in \operatorname{Prox}(i ; k)$ do

1.3.1 While $\left(\sum_{k \in K} n_{i}^{k}\right)-N_{i}>0$ and $N_{i}-\left(\sum_{k \in K} n_{j}^{k}\right)>0$ do Decrease units from $n_{i}^{k}$ and Increase them in $n_{j}^{k}$

3. If all the generating points satisfy condition $\sum_{k \in K} n_{i}^{k} \leq N_{i}$ then a solution to the problem has been obtained. Otherwise, a modification of parameters $R^{k}$ or $N_{i}$ is required.

\section{End.}

\section{COMPUTATIONAL EXPERIENCE}

Our model has been tested on a laboratory example, described by a graph representing a part of the street system in the city of Seville. The historic centre of Seville is one of the largest in Europe, along with those of Venice and Genoa. It has an approximately circular configuration and an area of $3.94 \mathrm{~km}^{2}$. The streets of this sector of Seville are predominantly narrow and one-way. The most common type of dwellings is the single-family house or the multi-family building with a shared courtyard. In both housing models, there is hardly any space available for the establishment of selective garbage containers in the interior. Conversely, citizens have traditionally orientated the common areas, both in the insides and doorways, towards decorations in tile and other traditional elements (gardening pots, etc.) that enhance the beauty of the place.

This unavailability of physical space for locating fixed containers forces to a widespread use of a small container (with a maximum capacity for 4 bags of garbage), mobile (provided with rear wheels and a handle to be dragged by the user), and polyvalent (without the possibility of classifying organic waste, glass, cardboard/plastic containers or paper), which serves the community of neighbours throughout each day.

The daily collection of waste from these multi-purpose containers is currently established by pre-designed truck routes, whose passing times are constrained to temporary windows that 
are known by the building neighbours. This collecting service is currently non-selective due to the above mentioned difficulties: widespread narrowness of streets to permanently locate containers in surface, aesthetic/tradition constraints, space limitations within dwellings that discourage the multiplicity of collecting instruments.

A computational experience has been carried out on a laboratory scenario composed of 39 dwellings (sites identified as elements of node set $I$ ). Location of nodes along the street network and the values of internode distances are illustrated in Fig. 1.

The amount of daily produced waste is randomised within the interval [400 kg, $16000 \mathrm{~kg}]$. By considering homogeneous containers of capacity $500 \mathrm{~kg}$, it is possible to initially assign to each residential place a container cluster (located at this same site) whose amount varies between 1 and 32. In order to provide the experiment a connection with a real context, a limitation on the number of containers that share the same geographical location has been set to 10. Additionally, a unit cost per locating a container at each site $j$ must be considered. In the experiment, it has been considered as randomised within the interval $[0,10]$, by representing the monthly cost in euros incurred on locating the container at this specific site.

In a first scenario, different types of garbage have not been distinguished. In Fig. 2, the container clusters, which are needed to guarantee the collection of all urban waste by means of user displacements to the deposit point nearer than $\mathrm{R}=100 \mathrm{~m}$, are represented by circles of variable radius between 1 and 10 .

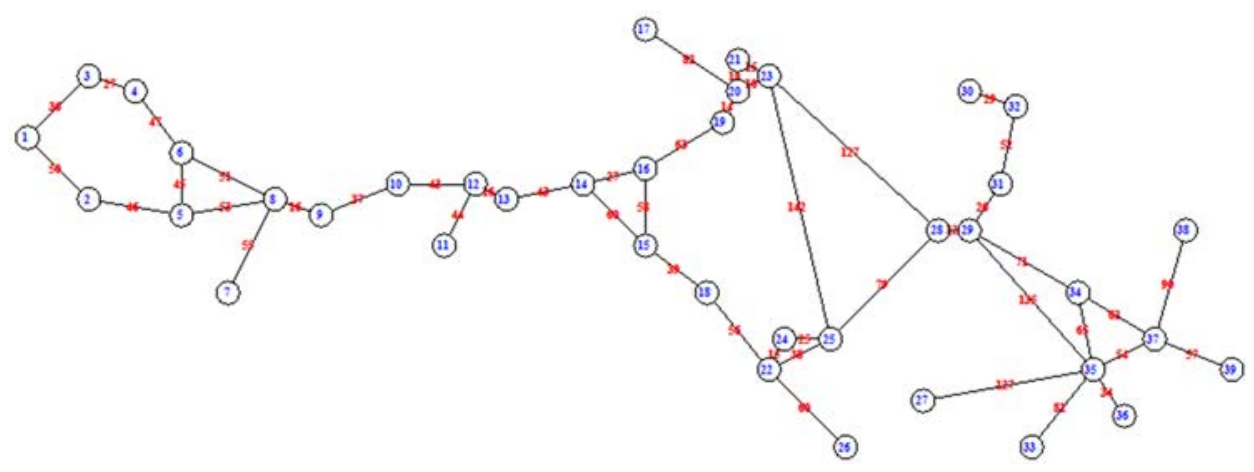

Figure 1: A network of thirty nine nodes.

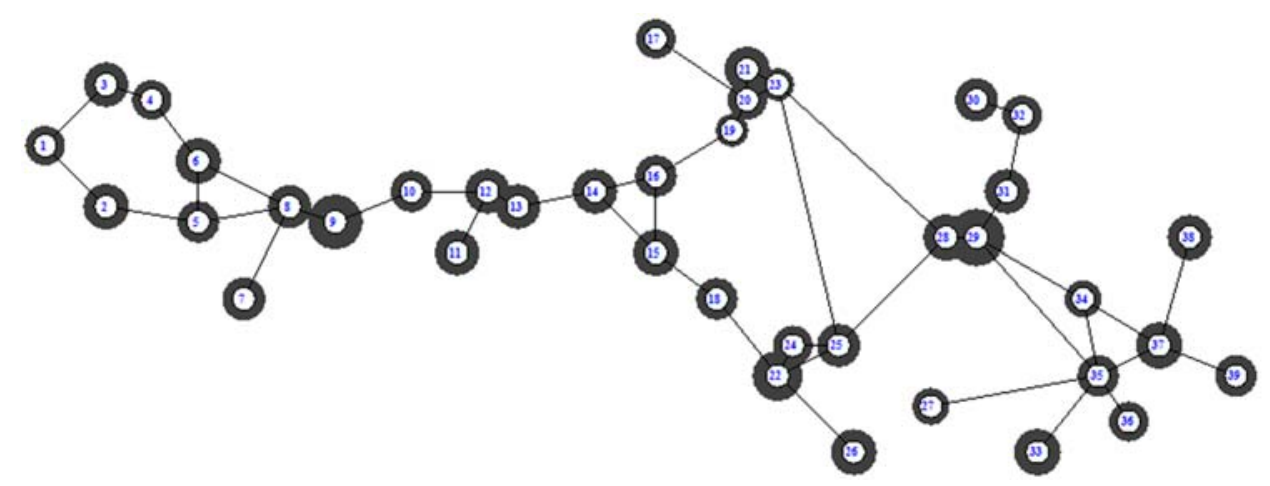

Figure 2: A garbage collection system with 39 nodes and a cost of 1091 euros per month. 
If no optimization procedure was applied, the 238 containers needed to collect all the global garbage produced (see locations in Fig. 2), would involve a cost of 1091 euros per month.

On the other hand, if we assume a solidary behaviour of the clients when taking their garbage to the container assigned to them within a pre-established proximity radius (not necessarily to the closest container to their place of residence), then a more efficient distribution of the containers can be obtained by means of the optimization model previously proposed. This optimized distribution is characterized by:

- A smaller number of clusters: the number of initial groups (39) has been reduced to 29.

- The compensation cost of the maintenance of the 238 containers on the street, is reduced by $14.48 \%$. The monthly cost associated to the solution shown in Fig. 3 is now 933 euros.

In a second scenario, we consider a selective collection of solid waste consisting of three different types (a situation like the one shown in Fig. 4).

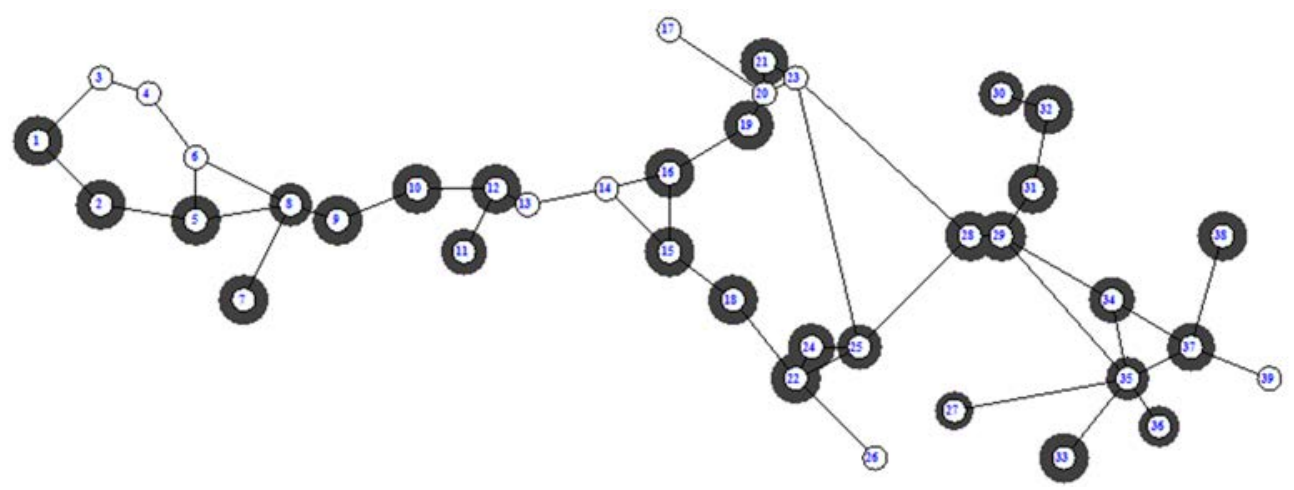

Figure 3: A garbage collection system with 29 nodes and a cost of 933 euros per month.

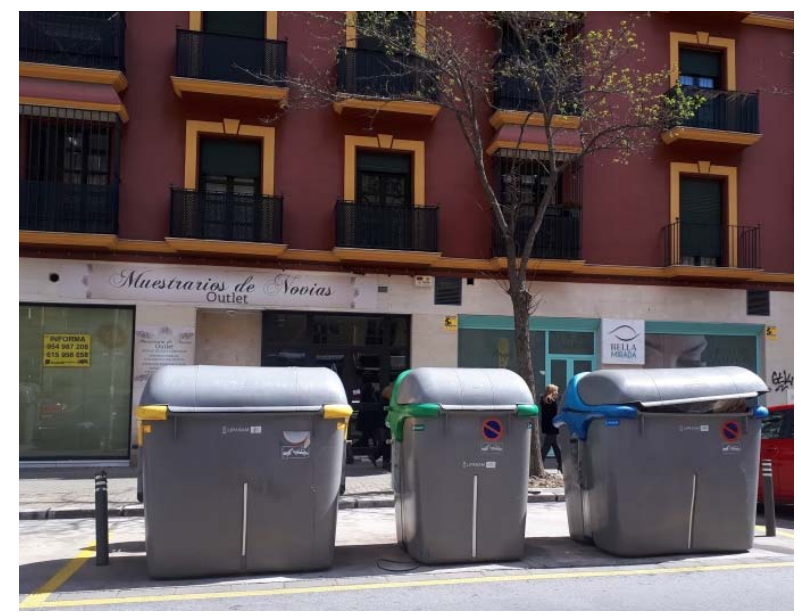

Figure 4: An example of three different types of solid waste collection. 
The total amount of waste generated at each node coincides with that of the first scenario, but its distribution in each of the three types of waste considered is random. The requirement to separately store the waste yields an increment in the number of containers needed to carry out the collection of waste. This increment with respect to the first scenario, which in the experiment is equal to $17.65 \%$ ( 280 containers now, versus 238 in scenario 1 ), leads to a redistribution of the containers. A new application of the ODIN heuristic, maintaining the values established for the parameters $R^{k}$ and $N_{j}$, provides the distribution of containers illustrated in Fig. 5. In this case, a more efficient distribution of the containers is also achieved after applying the algorithm, since those can be grouped in 33 places, instead of the initial 39 nodes.

If we compare the solution obtained in scenario 2 with the proposal for scenario 1 , we can observe that 17 nodes (marked with the magenta colour) have had to change their allocation of containers due to the increment caused by the selective collection of solid waste.

\section{CONCLUSIONS}

A methodology for the deployment of containers for selective collection of urban solid waste has been proposed in this work. The mathematical optimization model formulated for this purpose has been identified as a version of the Partial Set Covering problem, whose computational complexity motivates the use of heuristics to face large real-life scenarios. Following that recommendation, a greedy algorithm of overflowing deviated to the immediate neighbourhood has been developed to solve the proposed mathematical programming model.

To illustrate the performance of the developed methodology, a computational experience has been carried out on an urban system composed of 39 nodes with randomised data inspired in a zone belonging to the area of Seville (Spain). Two different scenarios are considered. The first scenario maintains the current non-selective collection of urban solid waste and, after the optimization procedure, the compensation cost is reduced by $14.48 \%$ and the number of clusters is reduced from 39 to $29(25.65 \%$ decrease $)$, thus facilitating the subsequent phases of service programming and collection route design. The second scenario incorporates the selective collection, thus yielding an increment on the number of containers but, even though, the number of nodes is reduced to 33 (15.38\% decrease). The evaluation of two generated scenarios illustrates then that the methodology meets the objective of efficiently designing a deployment of containers for selective collection of urban solid waste.

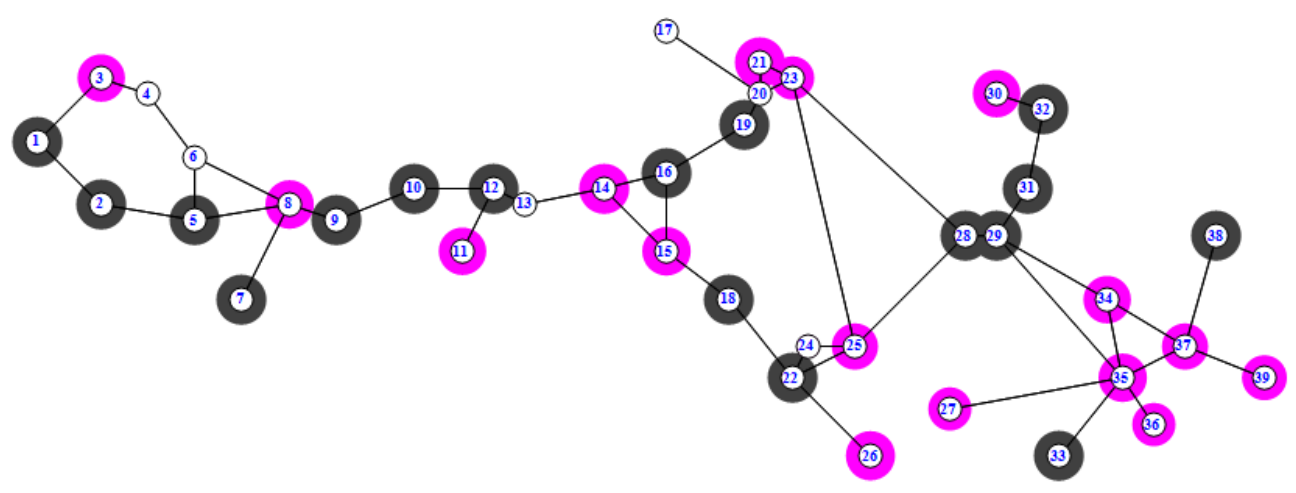

Figure 5: A garbage collection system with 33 nodes. 


\section{ACKNOWLEDGEMENTS}

This research has been partially supported by the Spanish Ministry of Science and Education through grants MTM2013-46962-C02-01 (MICINN, Spain) and MTM2016-74983-C2-1-R, and finally by the FEDER funds of the European Union. This support is gratefully acknowledged.

\section{REFERENCES}

[1] Khan, D. \& Samadder, S.R., Municipal solid waste management using geographical information system aided methods: A mini review. Waste Management \& Research, 32(11), pp. 1049-1062, 2014. DOI: 10.1177/0734242x14554644.

[2] Aremu, A.S., In-town tour optimization of conventional mode for municipal solid waste collection. Nigerian Journal of Technology, 32(3), pp. 443-449, 2013.

[3] Nuortio, T., Kytojoki, J., Niska, H. \& Braysy, O., Improved route planning and scheduling of waste collection and transport. Expert Systems with Applications, 30(2), pp. 223-232, 2006. DOI: 10.1016/j.eswa.2005.07.009.

[4] Eiselt, H.A. \& Marianov, V., Location modeling for municipal solid waste facilities. Computers \& Operations Research, 62, pp. 305-315, 2015.

DOI: 10.1016/j.cor.2014.05.003.

[5] Apaydin, O. \& Gonullu, M.T., Route time estimation of solid waste collection vehicles based on population density. Global Nest Journal, 13(2), pp. 162-169, 2013. DOI: $10.30955 /$ gnj.000739.

[6] Tung, D.V. \& Pinnoi, A., Vehicle routing-scheduling for waste collection in Hanoi. European Journal of Operational Research, 125(3), pp. 449-468, 2000.

DOI: $10.1016 / \mathrm{s} 0377-2217(99) 00408-7$.

[7] Ortega, F.A., Barrena, E. \& Canca, D., Improving Circulation Plans of Rolling Stock in Railway Networks by means of the Optimal Location of Depots. XXIII EURO Working Group on Locational Analysis, pp. 14-16, Sep. 2016. Malaga, Spain.

[8] Daskin, M.S. \& Owen, S.H., Two new location covering problems: The partial pcenter problem and the partial set covering problem. Geographical Analysis, 31(3), pp. 217-235, 1999. DOI: 10.1111/j.1538-4632.1999.tb00979.x.

[9] Cormen, T.H., Leiserson, C.E. \& Rivest, R.L., Introduction to Algorithms, MIT Press: Cambridge, MA, 1991.

[10] Barrena, E., Canca, D. \& Ortega, F.A., Routing vehicle fleets during disaster relief. Proceedings of the VIII International Workshop on Locational Analysis and Related Problems (2017), eds. M. Baldomero-Naranjo, I. Espejo-Miranda, L.I. MartínezMerino, A.M. Rodríguez-Chía \& D. Ruiz-Hernández, ISBN:978-84-697-5263-0, pp. 15-16, Sep. 27-29, 2017, Segovia, Spain. 